\title{
Laser Power Transmission and Its Application in Laser-Powered Electrical Motor Drive: A Review
}

Review

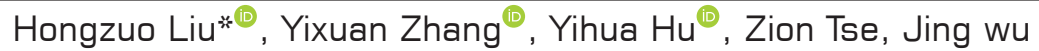

University of York, York, United Kingdom

Received: July 05, 2021; Accepted: September 13, 2021

\begin{abstract}
Laser power transmission (LPT) is considered a potentially efficient way for power delivery, especially in long-distance wireless applications and harsh hazardous environmental conditions. In contrast to other wireless power transmission (WPT) methods, LPT has many advantages such as lower device size, focused transmitting direction and high power density. With the development of technology, LPT has been widely adopted in several fields. In conservative industries, the utilisation of LPT can resolve the limitation problem in a wired connection. The adverse influence of electromagnetic interference (EMI) concerning application and high-temperature fields can be reduced. This paper will give a simple review of LPT and demonstrate the basic concept of a photoelectric emitter, transmission channel and receiver material. Based on the recent research about diode laser beam combining technology and high-efficiency multi-junction photovoltaics (PV) materials, the advised LPT devices for simple application as laser power motor will be simply discussed.
\end{abstract}

Keywords: Laser power transmission • Photovoltaic materials • Laser diode • Optical fibre power transmission • Atmosphere transmission

\section{Introduction}

Energy utilisation has become a part of our daily life. Especially, electricity is extensive energy, which has been widely utilised in transportation, communication, industrial production and aerospace. Transmission and consumption of energy have changed human production and lifestyle and promoted the development of society.

As the demand increases for recharge or other electricity transmissions, traditional wired-connection power delivery has gradually become less satisfying because of many disadvantages. The overload of the circuit can cause a disastrous fire in the building, which is dangerous to human life and property. Although some safety procedures can effectively cut off the electric circuit and prevent overload, the physical defects of the line circuit itself are difficult to achieve complete protection. Plug and unplug operation of line plug is inconvenient in multi-line connection fields. The complex interconnection increases the working difficulty of the connecting operator, and a mistake can bring unpredicted damages. Due to the physical factor such as high temperature in some industry application fields, such as nuclear stations, the copper wire connection can cause electromagnetic interference (EMI) to the working state. Moreover, wired connection power transmission is not suitable for long-distance transmission due to its high cost. To overcome these problems, a new mode of transmission is needed to break the limit while improving efficiency.

Wireless power transmission (WPT) is a potential method to avoid wire restriction. In general, some restrictions should be met to implement WPT: (1) can be transmitted in the atmosphere in an appropriate manner, (2) can do the directional transmission, (3) can convert energy sources into transmissible energy and (4) transmitted energy can be converted into usable and required energy types (Summerer and Purcell, 2009). There are different methods in WPT. Specifically, WPT methods can be classified into two types based on the transmission distance: nearfield transmission and far-field transmission. Based on the different power transmission mechanisms, WPT can 
be divided into four classes: capacitive power transmission (CPT), inductive power transmission (IPT), microwave power transmission (MPT) and laser power transmission (LPT). CPT and IPT are near-field techniques, and the other two are far-field techniques (Jin and Zhou, 2019). Considering that LPT has a long transmission distance and large power output (could reach 5 TW [Thul et al., 2021]), it is a suitable choice for WPT.

Laser power has been utilised in several novel areas such as weld and de-icing (Zhang et al., 2010). Because LPT has flexible workplaces and long-distance transmission capabilities, it can be used to transfer energy to drive the motors, thereby avoiding problems related to EMI and high-temperature-influenced problems of many applications such as deep hole drill in oil industries, aircraft and robotics. Laser devices have smaller sizes and are easy to implement. Besides, the transmission track of the laser is straight, media and easy to adjust the transfer route. There are two ways for laser transmission: one is tracking through the atmosphere; the other is an optical fibre. In atmosphere laser transmission, the power loss should be carefully considered. When transmitted through optical fibre, the power loss would gradually reduce when the transmission distance increased. Typically, optical fibres are made of materials that can withstand high temperatures, so LPT is suitable to be applied in harsh hazardous situations such as nuclear power plants and high-temperature oil pumps.

LPT has many limitations to overcome. The atmosphere is composed of different kinds of gases, such as nitrogen, oxygen and carbon dioxide. Therefore, if a laser travels through the atmosphere, there will be different absorption rates and energy losses (McComb et al., 2010). If an optical fibre is used to direct the laser energy, it can avoid the limitation of laser transmission in various media. It should be considered that one laser emitter might not guarantee enough output power. The recent diode laser-related research is mainly focusing on the multi-diode laser power beam combination technology, which combines the output beams of a set of diode lasers into a high-quality and high-power laser power beam to meet the output demand. Recent research on photovoltaic materials investigates materials with higher conversion efficiency, which also improves the overall system efficiency. The multi-junction Gallium arsenide (GaAs) photovoltaics (PV) materials have higher photovoltaic conversion efficiency than the singlejunction GaAs PV materials. Specifically, multi-junction GaAs PV materials can absorb different kinds of wavelengths and maximise the light power received. Both technologies are beneficial in improving the LPT system efficiency.

When the LPT system is used as an actuator, the main point of the research is focused on whether the existing LPT technology can meet the energy and efficiency demands of a motor drive. Therefore, it is necessary to review the LPT system and its components, which is also the focus of this paper. A general review of the LPT technology and the level of development will be given in this paper. This paper first introduces the background and application demand of LPT in Section 1. Section 2 will review the basic model of LPT and recent research progress on each part of LPT including a laser emitter, transmission channel characteristics and a PV receiver to discuss the optimal application selection. Section 3 will discuss and propose an LPT structure and discuss the feasibility of employing LPT for motor drive. Section 4 will list the future scope that shows the direction for improving LPT. The conclusion will be drawn for the possibility of realising LPT with the existing condition in Section 5.

\section{LPT Review}

The basic theory of LPT technology was proposed in 1965 based on the photoelectric effect principle. The structure of LPT is shown in Figure 1. On the left side, the laser power supply is used to generate a proper and steady

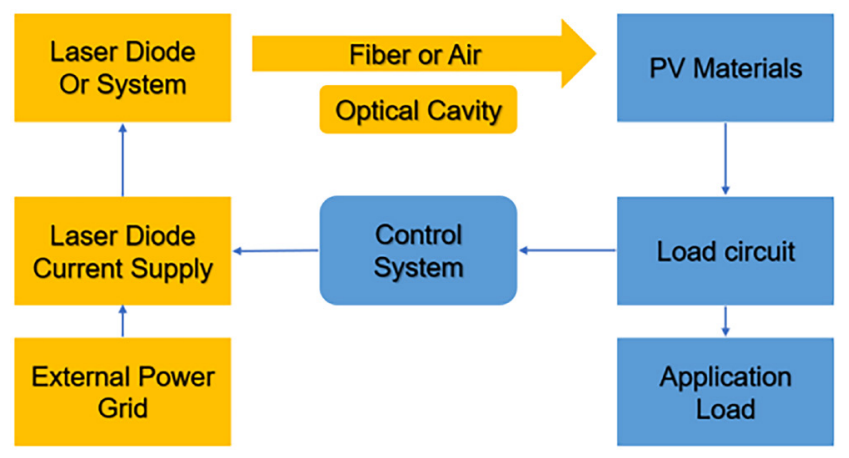

Fig. 1. Basic LPT structure. LPT, laser power transmission; PV, photovoltaics. 
current to the laser diode (LD). The LD converts electricity into a laser and then transfers it through the channel. The receiver consists of $\mathrm{PV}$, which are used for absorbing laser power beams to produce current. Besides, a power controller can be added to the system for rectification. All these processes are controlled by a control system (Jin and Zhou, 2019).

\subsection{LPT application history}

LPT is first proposed by NASA for aerospace wireless energy transmission. But due to the limitation of the low conversion efficiency in both LD and PV materials, the LPT is hard to be applied. In the 21st century, with the developed high-power LD and highly efficient GaAs PV, the LPT technology has gradually been applied. Many organisations did the LPT experiment and achieved different power delivery efficiency.

In 1985, Alejnikov et al. realised the fibre optic LPT, which applied $7 \mathrm{~W}$ in CO laser and $15 \mathrm{~W}$ in carbon dioxide laser (Alejnikov et al., 1985).

In 2002, the European Aeronautic Defence and Space Company (EADS) Space Transportation facility had an experiment about long-distance laser power transfer on the ground. The experiment used an LD named Nd:YAG, which had $5 \mathrm{~W}$ output power at the wavelength of $940 \mathrm{~nm}$. It provided approximately $1 \mathrm{~W}$ power for a small car distancing $250 \mathrm{~m}$. This experiment proposed the initial application of LPT technology (Steinsjek et al., 2003).

In 2003, NASA first powered a micro aircraft by LPT. In this experiment, the aircraft carried a PV material named $\mathrm{Ga}: \operatorname{lnP}_{2}$. The photovoltage conversion efficiency was $17.7 \%$ and the diode was $1.5 \mathrm{~kW}$ adjustable with $50 \%$ conversion efficiency. The PV provided $6 \mathrm{~W}$ of power to a $15 \mathrm{~m}$ high aircraft and made it fly for $15 \mathrm{~min}$ (Raible, 2008).

In 2006, Kinki University initiated the LPT-powered micro aircraft study. The chosen laser power was $300 \mathrm{~W}$. They achieved $25 \%$ transfer efficiency using the GaAs materials and converted $40 \mathrm{~W}$ power. The aircraft in their design can fly for $1 \mathrm{~h}$ at $50 \mathrm{~m}$ height (Kawashima and Takeda, 2005).

In 2008 and 2011, Raible in Cleveland State University introduced the relevant LPT technology, which provided theory support for the real application (Raible, 2011).

In 2009, the US Laser-Motive company experimented the feasibility of driving the elevator by LPT. The laser power was $1 \mathrm{~kW}$. Transfer distance was $1 \mathrm{~km}$ and total system efficiency was >10\% (Becker et al., 2010; AUVSI, 2012).

In 2011, Duke University began the research on LPT in the aspect of safety transmission. According to the IEC 60825-1, the maximum amount of laser radiation to the human body is relevant to time and wavelength. Commonly, shorter radiation time or higher wavelength is safer for the human body. Thus, Duke University selected 1,400 nm LD and Gallium Antimonide (GaSb) PV materials to form an LPT system successfully to supply power for a telephone in a $4 \mathrm{~m}$ distance (Sahai and Graham, 2011). Besides, Chongqing University built a laser network to supply power for a wireless sensor network. The selected 1,064 nm pumped LD can output $3 \mathrm{~W}$ power with $15 \%$ conversion efficiency (Wang, 2011).

In 2013, Tsinghua University proposed a feedback resonance method for laser power transfer. The reflected photon of PV arrays is used to stimulate the emitted photon again, which increased the total transmission efficiency from $4.7 \%$ to $6 \%$, comparing with no cavity (Huang, 2013).

In 2014, the Beijing Institute of Technology proposed a type of GaAs PV material that can afford 60 times standard light irradiation. Then based on this optimal PV material, a 100 m LPT experiment was carried out. The results indicate under the irradiation of $793 \mathrm{~nm}$ laser, the conversion efficiency of PV material was $40 \%$ for $24 \mathrm{~W}$ input laser power, and the total system transfer efficiency was 11.6\% (Bogachev et al., 2012). At the same time, Shandong Aerospace Electronic Technology Institute did a verification experiment for LPT to aircraft. The system employed LD with a wavelength of $810 \mathrm{~nm}$, laser power of $28 \mathrm{~W}$ and the maximum transmission distance of $200 \mathrm{~m}$ to achieve the $15 \%$ of the total system transfer efficiency (Shi et al., 2016).

In 2016, Russian Energy Rocket Space Group Co. Ltd successfully charged one telephone $1.5 \mathrm{~km}$ away. The experiment device converted laser power into electrical power with 60\% transfer efficiency (Mohammadnia et al., 2021). Besides, Dele Shi et al. in Shandong Institute of Aerospace Electro-technology conducted an on-board experiment on the LPT subsystem. The transmission distance was from $50 \mathrm{~m}$ to $100 \mathrm{~m}$, and the input voltage, current and power were $22 \mathrm{~V}, 4 \mathrm{~A}$ and $88 \mathrm{~W}$, respectively. The result shows that the received power was $13.43 \mathrm{~W}$ and the maximum efficiency was $16.08 \%$ (Shi et al., 2016).

Recently, in 2021, Mohammadnia et al. studied LPT technology in driving next-generation drones. The PV materials were equipped on the drone to receive laser power. The experiment had adopted three different types of 
PV materials: GaAs, CdTe and c-Ti. They received the power of $73.5 \mathrm{~W}, 62.6 \mathrm{~W}$ and $33.2 \mathrm{~W}$, respectively. In the transmission distance of $500 \mathrm{~m}$, it had a total power transfer efficiency of $12.5 \%$ (Mohammadnia et al., 2021).

From the above research, there has been the application supplied by the existing LPT technology. However, the system energy transmission efficiency is generally low. The conversion efficiency of LD and PV materials is the main reason that influences the total system power delivery. However, improving transmission efficiency is still the key breakthrough direction of the LPT system for supplying sufficient and stable current to the load.

\subsection{Laser Emitter}

Laser emitter is playing a role in converting electrical power into laser power in LPT technology. The most common type of laser emitter is LD, which uses the photogenic volt effect of the PN junction to work. The output power efficiency of LD is changed by changing the supply current of the laser power source. The power efficiency of one $975 \mathrm{~nm}$ LD coupled with fibre optic is shown in Table 1. The output power and conversion efficiency of LD are often increased with the supply current raise, but the raised speed of conversion efficiency will decrease. The experiment results shown in the literature (Zhou and Jin, 2015a, b) have proved that the LD supplied by pulse wave current can achieve better efficiency than that supplied by constant current. As shown in Figure 2, the power conversion efficiency will be higher when the duty cycle $D$ is lower.

Recent research has shown interest in the diode laser combined technology. In this method, all the outputs of every single LD are combined into one laser power beam, which obtains high brightness, high beam quality and high power density. For the combined output laser power beam, it ensures to satisfy the demand of power for terminal machines that a single LD cannot meet. The different systems may have different power perimeters and designed power structures. The output power of these examples is varying from watts to kilowatts. These studies are reviewed as follows:

There was much research done in 2013. Röhner et al. present the radiance model of LD and how the radiance influenced the power density. The result shows that the laser beam combining technology does not increase the radiance more than the initial value. The example used a $200 \mathrm{~W}$ system model with a $7+1$ combined source to pump $400 \mu \mathrm{m}$ fibre (Röhner et al., 2013). Zimmer et al. have researched the direct diode laser system which can reach the output power for several kilowatts by applying beam combining technologies. As a result of using this on the low-power laser bars for 6-7 W each, the beam quality has improved by $30 \%$. After that, they improved the laser bar output for $120 \mathrm{~W}$ and combined 12 such laser bars at $1.4 \mathrm{~kW}$ for the output of the system and the conversion efficiency was $>50 \%$ in this process. Finally, they designed a $6 \mathrm{~kW}$ direct diode laser TRU-Diode 6006 which can match the output of $4-5 \mathrm{~kW}$. This research has proved the spatial and spectral beam combining technology can

Table 1. The power of $975 \mathrm{~nm}$ fibre coupled with LD (Song, 2018)

\begin{tabular}{lrrrrrrrr}
\hline Current J/A & 2 & 3 & 4 & 5 & 6 & 7 & 8 & 9 \\
\hline \hline Laser power P/W & 7.6 & 12.8 & 18.0 & 22.6 & 27.3 & 31.8 & 35.9 & 39.8 \\
Laser power density $\mathrm{mW} / \mathrm{cm}^{2}$ & 26.5 & 44.6 & 62.8 & 78.8 & 95.2 & 110.9 & 125.2 & 138.8 \\
\hline
\end{tabular}

LD, laser diode.

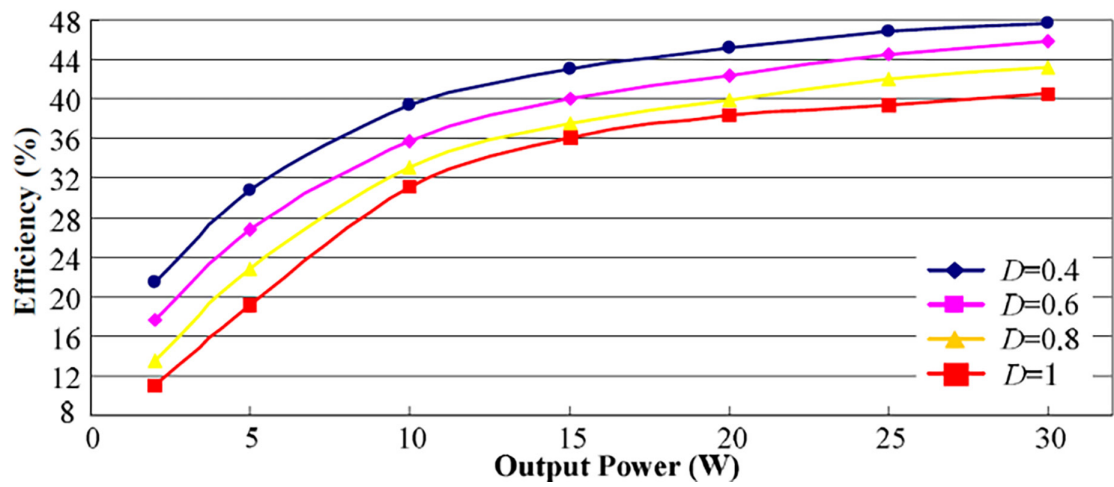

Fig. 2. Efficiency of LD powered by pulse wave current supply (Zhou and Jin, 2015a, b). LD, laser diode. 
realise high-brightness multi-kilowatt output laser beam for high-command applications from a single low-power laser emitter (Zimer et al., 2013). Unger et al. described a project which aimed to build a direct diode laser system to reach the output of $>2 \mathrm{~kW}$ and efficiency of $>40 \%$ for industry applications. If constructed by carbon dioxide lasers, the efficiency can be no $>20 \%$. Fibre laser was grown well in such applications, for it can realise the optical conversion efficiency of $80-85 \%$ and the total power efficiency of $30-35 \%$. In a block of the BRIDLE, the single emitter was up to $10 \mathrm{~W}$ and the total output for this block was $120 \mathrm{~W}$ (Unger et al., 2013). Brand et al. designed a platform of diode lasers for industrial application at the multi-kilowatt level. The system fibre laser source pump needed $200 \mathrm{~W}$ power and it had seven laser bars to combine the one output source. In such a system design, they adopted NA 0.22 fibre, which is only $200 \mu \mathrm{m}$. To couple with this fibre, they built a base subunit that contained five laser minibars, which satisfied the demand of shape, size and laser amount. This unit can give the output for up to $260 \mathrm{~W}$, with the current of $40 \mathrm{~A}$ in central wavelength at $976 \mathrm{~nm}$. The photovoltaic conversion efficiency was $52 \%$. To design a high-power system, the system combined with four base subunits was at $800 \mathrm{~W}$ in a central wavelength of $976 \mathrm{~nm}$. For eight base units combined system, the output can reach 1,500 W. This system can work at five modules in different central wavelengths, which were $915 \mathrm{~nm}, 940 \mathrm{~nm}, 980 \mathrm{~nm}, 1,020 \mathrm{~nm}$ and 1,060 nm. If the current was $45 \mathrm{~A}$ for each laser minibar, the output could reach $4 \mathrm{~kW}$ (Brand et al., 2013). Helal et al. did a simulation on the laser beam propagation routes and characteristics in the large cavity. They combined several LDs to reach a total output of 100-1,000 W. The basic structure of the high-brightness system was described. The system contained several diode lasers, which can be single or a group of LDs or laser bars. Before the output laser power beam of every single emitter was combined by optical devices, they needed to be collimated with optical collimation devices. In a high-power system, a large volume of the cavity was needed. The output of the beam assembly phase must be ultimately focused on the fibre through further optics. The simulation process was carried out by the software instruments such as Zemax and Speclase. The result showed that most of the beams were coupled into output and some were back to the LD, and the scattered ray remained was the power loss. The challenge was converting the feedback laser power beams into utilisation to reduce the power loss (Helal et al., 2013). Witte et al. demonstrated a type of high-brightness diode laser module which is used for pumping applications at the red spectral range. Different types of solid lasers were made of different materials, which needed different beam wavelengths of the laser source. As mentioned, the Nd:YAG needs $808 \mathrm{~nm}$, the Yb:YAG $941 \mathrm{~nm}$ and the Yb3+ doped silica $975 \mathrm{~nm}$, respectively. Seven single emitters were stacked vertically in a ladder shape. The block can reach an output of $15.5 \mathrm{~W}$ with the optical-to-optical conversion efficiency of $>95 \%$. The factors of power loss were mainly the diffraction caused by lens aperture and the reflection by the surface of the lens (Witte et al., 2013). From the research by Stefan Hengesbach et al., DFB/distributed Bragg reflector (DBR) diode laser module designed by spectral multiplexing was the most efficient way which was built on VBGs. The combined multiplexing could reach $97 \%$. There were two concepts of the multiplexing method mentioned. One was dense spectral combining technology, which limits the spacing of the centre wavelength to $4 \mathrm{~nm}$. The other was wavelength beam combining technology, which combines the stable frequency in the external cavity with the diffractive element. The system was designed based on BRIDLE-Project. Five emitters were made one group of bars. The result showed that the multiplexing efficiency in single mode was $97 \%$ and in multi-mode was $85 \%$ (Hengesbach et al., 2013). The cavity was the important part of the diode laser to deal with the problem of feedback laser power beams. Super-largevolume cavity studied by Bull, Kaunga-Nyirenda and Larkins showed how to design this external cavity of diode lasers. As mentioned, both spectral beams combining and coherent beam combining could be adopted in this application to produce a high-power and good-quality beam, which duly handles the feedback into the laser. They concluded that not all the LD was suitable for equipped a cavity. Larger was better but would be something wrong in high-order mode. The system stability and efficiency should be considered when designing (Bull et al., 2013).

In 2015, Dan McCormick et al. showed a type of diode-pumped alkali-vapour lasers, as the DILAS has grown a source of a LD which outputs $2.5 \mathrm{~kW}$ of fibre optical power at an almost central wavelength at $766.5 \mathrm{~nm}$. The $90 \%$ was distributed in the power enclosed spectral linewidth. Also, the central wavelength could be controlled in a range of $\pm 0.15 \mathrm{~nm}$. Such technology has been applied in fields for different wavelengths and styles, and the power varied from hundreds to thousands of watts (McCormick et al., 2015). There was one type of fibre-coupled diode laser demonstrated by Ulrich Witte et al. The output could reach $10 \mathrm{~W}$ and up to $100 \mathrm{~W}$. A method called dense wavelength division multiplexing (DWDM) was applied to combine a 5-emitter narrow stripe broad area mini-bar. It used fibre combiner and coarse wavelength beam combining to design this system. In such a designed system, the current supplied to injection was $40 \mathrm{~A}$ and the measured optical power was $53.2 \mathrm{~W}$. The $32.2 \mathrm{~W}$ was the output power produced by DWDM. The calculated combined efficiency was $60 \%$ (Witte et al., 2015). The process of 
high-brightness direct diode laser at kilowatts level has been researched by Huang et al. The first generation of lasers was carbon dioxide and YAG lasers, which had a small size. As for the second generations, they were fibre laser and disc laser and had the benefit of better efficiency. And the third generation was the direct diode. The system power conversion efficiency was $44 \%$ under the power of $4 \mathrm{~kW}$, at a driving current of $180 \mathrm{~A}$. The max current can be $220 \mathrm{~A}$, and the power can reach 4,680 W (Huang et al., 2015). Zimer1 et al. researched a thin-film filter for dense wavelength beam combining technology. The result showed that the output obtained was $550 \mathrm{~W}$ with electrical to the optical power conversion efficiency of $40 \%$ above, which was $10 \%$ lower than free direct LD. Compared to solid pumped lasers, the direct LD had better conversion efficiency and lower cost (Zimer et al., 2015). There was another high-power and high-brightness diode laser system in multi-kilowatt level presented by Rossin et al. They adopted broad area diode lasers at a power conversion efficiency of $50 \%$ with fibre-coupled modules. The conclusion showed that the improvement can reach $30 \%$ of the current generation model for multi-emitter mode (Rossin et al., 2015).

The DBR ridge waveguide (RW), which had wavelength stabilised for special pumping application, was demonstrated by Bernd Sumpf in 2016. The tapered laser was the famous way to produce high-power low-diffraction limited beams. For DBR tapered lasers, they had $12.2 \mathrm{~W}$ output with $40 \%$ conversion efficiency at $25^{\circ} \mathrm{C}$ under a wavelength of 1,064 $\mathrm{nm}$ (Sumpf, 2016).

In 2017, Klaus Kleine and Prabu Balu proposed the materials processing powered by the high-power diode laser system. When using solid-state pumped laser systems, the high-power diode lasers were utilised as the pump source. Over $90 \%$ of the diode lasers' output was going for the pumping process of the solid lasers and the last small part of the power was directly for materials processing. They combined these emitters or several groups of emitters into one fibre to increase the total output. In the system they were mentioned, a type of stack which was consisted of several laser bars can reach outputs of 50-400 W. Then, the combined laser power beam of these laser bar stacks can realise the output power in kilowatts (Kleine and Balu, 2017). Meanwhile, Lucas-Leclin et al. discussed the coherent beam combining technology. In on-axis diffracted order, the maximum output power was $7.5 \mathrm{~W}$ with the beam combining efficiency of $65 \%$. For the power amplifier, five tapered laser bars were combined in a group. The results proved that the beam combining efficiency was $82 \%$, at a supply current of $3 \mathrm{~A}$, and it can reach $72 \%$ in the maximum current of $6 \mathrm{~A}$. The maximum output power was $11.5 \mathrm{~W}$ (Lucas-Leclin et al., 2017). Laser was the new tendency of the way for lighting, replacing existing LED.

In 2019, Anastasiia krasnoshchoka studied two different LDs for generating white light. For power concern, the single broad area LD can have an output of $3.5 \mathrm{~W}$ at a wavelength of $445 \mathrm{~nm}$. The other was a group of blue diode lasers with eight inside. The output can reach $35 \mathrm{~W}$ at $450 \mathrm{~nm}$. The spot size can be adjusted (Krasnoshchoka et al., 2019).

From all the research listed above, the output of the current supply will influence the power efficiency of the LD. If the LD works in a high-pulse and low duty cycle state, the power requirements are also very high, especially in accuracy and stability. That will be one limitation of the efficiency improvement. However, the use of efficient power supplies also contributes to the efficiency improvement of LD. Laser power combination technology can be an effective and feasible method to improve the total output power and to meet the demand of the terminal load. The combination efficiency is generally acceptable for the application. But combination system design will increase the complexity of devices, which will cause inconvenience for use. Diode lasers can be considered when a single LD power is insufficient. The challenge of LD is the development of manufacturing technology.

\subsection{Transmission through the atmosphere}

The wavelength between $3 \mu \mathrm{m}$ and $4 \mu \mathrm{m}$ or $8 \mu \mathrm{m}$ and $14 \mu \mathrm{m}$ is transparent, but laser transfer efficiency in the respective region is low. The range of $\sim 780$ to $1,100 \mathrm{~nm}$ can be the window for existing laser power devices to transfer power adequately, as shown in Figure 3.

The observed result of LPT in the atmosphere was studied in the literature (McComb et al., 2010) along with moderate-resolution atmospheric transmission (MODTRAN) code-based simulations. The data is collected maximum at $2,050 \mathrm{~nm}$. This result shows the transparency window from $2,025 \mathrm{~nm}$ to $2,100 \mathrm{~nm}$, and $<1,960 \mathrm{~nm}$, the power will be strongly absorbed. This experiment was limited to low-power circumstances between $1 \mathrm{~W}$ and $10 \mathrm{~W}$.

Solar energy outside the earth is attractive to make electricity production. Rubenchik et al. (2009) studied the self-focusing of the laser beam in the atmosphere, which helps to assist in delivering powerful laser beams. If the power of beams exceeds the standard self-focusing power, the uncontrolled beams may cause disaster for devices. 


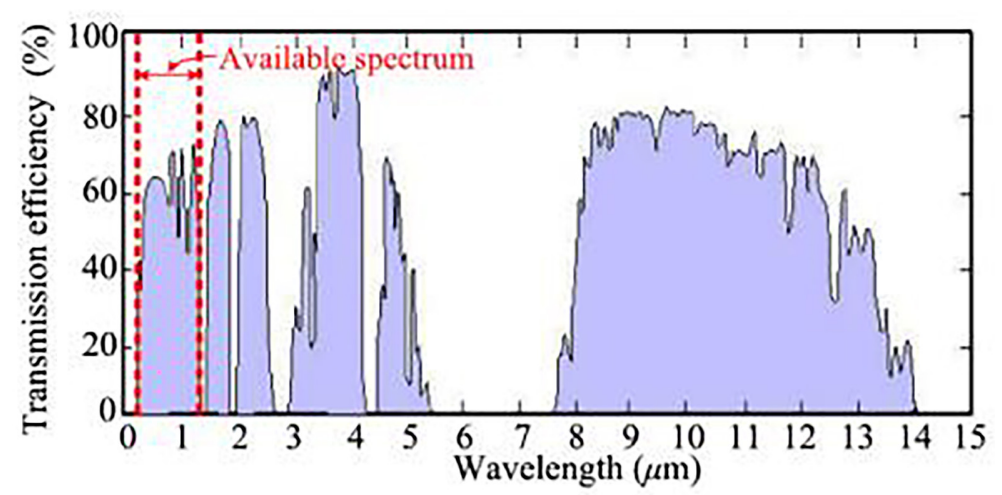

Fig. 3. Atmosphere transmission wavelength absorption (Jin and Zhou, 2019).

When the laser beam is transmitted to the target, a portion of the energy is absorbed by the atmosphere (Yao, 2009). There are two important influences for LPT. One is the power intensity of the laser will be weaker, which results in a reduction in transmission efficiency. The other more important factor is that the atmospheric absorption will heat the channel for LPT. The shape of the laser beam will get distorted, which creates the thermal halo effect. This will further increase the divergence of the laser beam and reduce the intensity. The relationship between the thermal halo effect and the laser power intensity distribution is nonlinear.

When the laser tracks through the air, atmospheric gas molecules and aerosol particles will absorb and scatter light waves, and then cause the attenuation of laser energy. Laser energy absorbed by the atmosphere per unit volume can be expressed as in Eq. (1):

$$
\varepsilon(z)=\alpha l(z)=\left(\alpha_{m a}+\alpha_{p a}\right) l(z)
$$

$\alpha_{m a}$ and $\alpha_{p a}$ are the gas absorb coefficient and the aerosol particle absorb coefficient, respectively. I(z) is the light intensity. The attenuation of the laser caused by absorbing and scattering can be generally presented by the deer theorem. Its differential expression is given in Eq. (2):

$$
d l(z)=\alpha_{t}(z) d z=\left(\alpha_{m a}+\alpha_{m s}+\alpha_{p a}+\alpha_{p s}\right) d z
$$

$\alpha_{m s}$ and $\alpha_{p s}$ are the gas scattering coefficient and the aerosol particle scattering coefficient, respectively.

The relationship between the wavelength and absorption is very complex and irregular. A suitable wavelength selection for laser power transfer is important. In addition, the absorption coefficient is constantly changing along the propagation path. When using LPT for short distances, air transmission will be very convenient if nothing stands in the transmission route. Otherwise, laser energy will attenuate as the distance increases. The propagation path will also be offset due to refraction. The challenge is to overcome the power loss and transmission route bending as the distance increases.

\subsection{Transmission through optical fibre}

The key to the realisation of optical fibre energy transmission technology is the energy transmission fibre. It has the characteristics of low transmission loss, high damage threshold and high-temperature resistance. Thus, it can reliably transmit energy in all kinds of extreme environments. When designing the transmission system, the input laser power efficiency, the fibre optical material and core diameter will be considered. The energy attenuation should be measured. A high-energy-density laser beam will cause damage to the optical fibre's end face. Two aspects are considered in the literature (Xiao, 2017): (1) Optical fibre material choice: optical fibre materials with low scattering loss should be selected. The material which contains transition metal and $\mathrm{OH}$ group has small absorption loss and stable material structure. (2) Optical fibre structure: The design of a large core diameter is beneficial to reduce the laser power density inside. 
The core diameter of single-mode fibre is approximately $2-12 \mu \mathrm{m}$. When the laser is transferred in high power density, the larger size core diameter will be used, because it will influence the performance and life of fibre optic. The laser damage threshold was measured at US Sandia National Laboratories (Zhao, 2007). The result was $215 \mathrm{~J} / \mathrm{cm}^{2}$ that processed by carbon oxide and was $185 \mathrm{~J} / \mathrm{cm}^{2}$ that not processed by carbon oxide. Reng and Beck (1993) discussed the limitation of the optical fibre transmission that depends on the parameters of the laser power beam, including laser beam diameter and divergence angle. When the laser beam is transferred through the fibre, the beam diameter shall be smaller than the fibre diameter. The divergence angle shall not be too big, for the beam reflection of the cladding and end face would cause crosstalk to the beam quality.

However, in high-radiation environments, radiation-resistant optical fibres made by special processes must be used to ensure transmission quality. The radiation emitted by nuclear waste can affect the transmission characteristics of optical fibre. This is due to the damage caused by high-energy radiation to the chemical structure of the optical fibre. When a fibre-optic transmission is used in a nuclear waste machine, radiation-resistant fibre is needed to ensure transmission quality. Loss and dispersion are the main parameters for the characterisation of optical fibre performance. The loss can directly determine the attenuation of lightwave energy (Liu, 2016). The main component of common quartz fibre is silica. When irradiated, the lattice structure of the optical fibre will change. Both the physical and chemical properties of the optical fibre are changed by defects in the lattice. There are three mechanisms that cause changes in optical fibre performance: (1) radiation-induced absorption (RIA), performing as the absorber of the transmission light by the fibre, (2) radiation-induced emit (RIE), performing as irradiated optical fibres produce light waves in certain wavelengths and (3) optical density (the refractive index of the irradiated fibre changes). Among these three mechanisms, RIA is considered the main cause of fibre performance decline (Zhang, 2017). These damages will greatly produce the loss of optical fibre. Moreover, the diameter of fibre determines the radiation loss. The experiment shown in the literature (Zhang, 2017) indicated that with an increase in diameter, the loss would decrease. And if the wavelength is increased, the loss would reduce.

The methods to improve the resistance radiation performance of optical fibre mainly include resistance radiation design of optical fibre waveguide structure, proper doping of fluorine element, effective control of manufacturing process, application of peroxide doping technology, pre-irradiation treatment and post-irradiation treatment technology application (Zhang et al., 2020). Literature (Wen, 2020) proposed a specific way to increase the resistance radiation of fibre. First, pre-irradiate the fibre to destroy the part of the easily activated free radicals, atomic defects or loose bonds in fibre, and bond it to a stable bond at high temperature. Then by light-fading and hydrogenation process, restore or repair the induced absorption caused by the structural defect of the optical fibre. Finally, deuterium gas treatment is used to reduce the additional loss caused by hydrogenation. Light-fading treatment mainly refers to that under high-intensity laser irradiation. The defects centre of a substance absorbs one or more photons, which will produce free electron heating and self-focusing effects, thereby improving the defect structure of the substance. The low-temperature treatment of liquid nitrogen is mainly to reduce the activation energy of the material, make the molecular arrangement of the material more orderly and reduce the number of internal defects in the material. The experiment process is to intercept the $2.8 \mathrm{~m}$ polarisation-maintaining optical fibre (PMF) after the pre-irradiation treatment of $1.0 \mathrm{kGy}$, and then use a monochromatic laser light source with a wavelength of $980 \mathrm{~nm}$ and output power of $100 \mathrm{~mW}$ to irradiate for $30 \mathrm{~min}$. Next, treat the optical fibre with liquid nitrogen for approximately $10 \mathrm{~min}$. After the polarisation-maintaining fibre is pre-irradiated with $1.0 \mathrm{kGy}$, its loss is greatly increased. Then, through the $980 \mathrm{~nm}$ laser light-fading treatment on the irradiated fibre, its spectral loss increases a lot, but the signal-to-noise ratio of its loss spectrum is obviously better. Then, after the liquid nitrogen treatment, its spectral loss will be greatly reduced, and it is lower than the pristine PMF spectral loss. It shows that the method improves the microstructure of the material and effectively reduces the loss.

Dropping to change the crystal structure of the fibre can also improve the resistance to radiation. Literature (Zotov, 2008a, b) have studied the radiation resistance of erbium-doped fibre. The optical fibre doping can change the refractive index of the optical fibre, thus changing the transmission characteristics of the optical fibre. By erbium doping combined with high-pressure hydrogen treatment, the RIA is reduced in the experiment measure result. Different doping also results in different radiation resistance. Literature (Billat et al., 2017) tested the germaniumdoped (Ge-doped) multi-mode fibre and the fluorine-doped (F-doped) radiation-resistant standard multi-mode fibre. The experiment result indicated that the F-doped fibre has obvious effectiveness in light fading than conventional Ge-doped fibre.

Something else worth mentioning is the colour centre, which is produced when the electron energy is higher than the atomic dislocation threshold in silicon dioxide. According to previous studies, the total dose of ionisation 
damage effect will not cause permanent damage to the optical properties of the fibre. At room temperature, after the irradiation is stopped, the colour centre of the fibre fades after a period of time. There are many high-energy defect structures in silica fibre. These high-energy defects often form colour centres after being irradiated. The electronegativity of fluorine is the strongest (stronger than oxygen). Doping with fluorine can produce a Si-F bond in the fibre, increase the probability of breaking the network of high-energy defect structures and reduce the defect concentration in the quartz glass. The light-fading has the opposite effect of radiation, to fix the defects in the lattice. During the process, the light power is higher and the effect of light fade is higher. And the function to reduce radiation loss is more effective (Zhang et al., 2020).

When laser power is transmitted through the optical fibre, the route could be organised in a proper way and the power loss would be lower than atmosphere transmission. But the flexibility in use will be reduced. Especially in high-radiation and high-temperature environments such as nuclear stations, the radiation resistance of fibre will directly influence the transmission power quality. The challenge is how to reduce the power loss in long distances and increase the radiation resistance.

\subsection{PV materials receiver}

A suitable photovoltaic conversion material is very important for absorbing laser energy and improving system efficiency. There are hundreds of materials developed, but the most common photovoltaic materials are Si and GaAs. The main parameters are shown in Table 2. Silicon is a very common substance, and its production cost is low. But the transfer efficiency is also low due to its sensitivity to the $950 \mathrm{~nm}$ wavelength, and the $950 \mathrm{~nm}$ is not sufficient to deliver power through the air. Because of the breakthrough of the photoelectric transfer rate of GaAs PV materials, the laser power transfer energy can have rapid developments (Zhuang et al., 2014). The $810 \mathrm{~nm}$ is the best power efficiency of GaAs, which is suitable for LD's wavelength of $860 \mathrm{~nm}$. During the process of the photoelectric transfer, the power loss includes LPT loss and photo-electric transfer loss. Table 3 has shown the power transfer efficiency in different PV materials. The material with a large bandgap has a high upper limit of conversion efficiency, but the loss in optical fibre transmission is larger. This is contradictory to material selection.

GaAs is a typical III-V compound semiconductor material, although it has the same sphalerite crystal structure as silicon. However, unlike silicon with an indirect bandgap, $\mathrm{GaAs}$ is a direct bandgap material, and it has a bandgap width of $1.42 \mathrm{eV}$, which is the best bandgap width that PV materials need. Because GaAs has the best power conversion efficiency that promotes the development and practical application of the LPT system, it has a very outstanding advantage in the application: (1) Direct bandgap structure: GaAs has large light absorb factor; it can quickly absorb the incident light. GaAs can achieve a lower size of devices, for to absorb $95 \%$ of sunlight, it only needs $\sim 5$ to $10 \mu \mathrm{m}$, which is just $10 \%$ of that required for silicon materials. (2) Good radiation resistance: The lifetime of minority carriers produced by direct bandgap GaAs is short. Especially in aerospace, the influence of

Table 2. Main parameters of PV materials (Zhou, 2018)

\begin{tabular}{lccc}
\hline Materials & Absorb peak wavelength & Efficiency $(\%)$ & Radiation intensity $\left(\mathrm{kW} / \mathrm{m}^{2}\right)$ \\
\hline \hline GaAs & $810 \mathrm{~nm}$ & 40.4 & 60 \\
& & 53.4 & 430 \\
& & 60 & 110 \\
$\mathrm{Si}$ & $950 \mathrm{~nm}$ & 28 & 110 \\
\hline
\end{tabular}

PV, photovoltaics.

Table 3. The relationship between material bandgap and cut-off wavelength and transmission loss (Zhuang et al., 2014)

\begin{tabular}{lccccc}
\hline Materials & Eg (eV) & $\begin{array}{c}\text { Cut-off } \\
\text { wavelength }(\mathrm{nm})\end{array}$ & $\begin{array}{c}\text { The upper limit of single- } \\
\text { junction conversion rate }(\%)\end{array}$ & $\begin{array}{c}\text { Losses in standard } \\
\text { optical fibres (dB/km) }\end{array}$ & $\begin{array}{c}\text { The light intensity } \\
\text { decays to 10\% (km) }\end{array}$ \\
\hline \hline GaAs & 1.42 & 873 & 60 & 2.5 & 4 \\
$\ln _{0.35} \mathrm{Ga}_{0.65}$ As & 0.95 & 1,310 & 45 & 0.5 & 20 \\
$\ln _{0.47} G_{0.53}$ As & 0.74 & 1,550 & 35 & 0.2 & 50 \\
$\ln _{0.7} G_{0.3} A s$ & 0.55 & 2,200 & 22 & 2.2 & 5 \\
\hline
\end{tabular}


GaAs is lower than that of Si. (3) High-temperature resistance: GaAs materials have a lower temperature coefficient and can adapt to higher working temperatures. (4) High photovoltaic conversion efficiency: Because GaAs has a wider bandgap and better match the sunlight (Han, 2018).

The power conversion efficiency improvement is the main development direction of PV materials. Many institutions have made progress in this direction. A three-junction gallium arsenide solar cell developed by Spire Semiconductor achieved a peak efficiency of $42.3 \%$. The latest ground-based solar cell, the C3MJ+, developed by Spectro-lab, a Boeing company, has an average photoelectric conversion efficiency of 39.2\%. Japan's Akita University developed an organic material that can convert ultraviolet light into visible light and is transparent to visible light. It used ultraviolet light that solar cells cannot effectively use for photoelectric conversion, thus improving the efficiency of photoelectric conversion. Wake Forest University in the United States increased the amount of sunlight collected by covering the polymer matrix of the cell with a vertical layer of optical fibres as a sun-trapping device. This layer of fibre protrudes from the surface. Sunlight can enter the top of the fibre from any angle, and photons bounce around the inside of the fibre until they are absorbed by the surrounding organic cells. Genie Lens, a US company, developed a polymer film with an embossed microstructure that redirects incoming light and increases its chance of being absorbed. The film can be simply placed on the surface of solar panels to increase their output power. Stanford University has developed a simple and inexpensive way to create large areas of nanoscale-textured structures. Researchers precipitated metal and amorphous silicon onto uneven surfaces to create superhydrophobic surfaces and proof-of-concept solar cell devices. The solar cell absorbs $42 \%$ lighter than a flat surface using the same amount of material (Anon, 2011).

Some research which aim at improving the power conversion efficiency of the PV materials concluded in (Han, 2018): Yugami et al. chose an $808 \mathrm{~nm}$ diode laser with an output power of $1 \mathrm{~W}$ radiation on a single-junction singlechip GaAs material. In the area of $4 \mathrm{~cm}^{2}$, the power receipted is $5 \mathrm{~W}$ with transfer efficiency could reach $51 \%$ (Yugami et al., 1997). Steinsiek chose the Nd: YAG with $532 \mathrm{~nm}$ wavelength and $5 \mathrm{~W}$ output power to drive the small car equipped with an InGaP material. The transmission distance was $300 \mathrm{~m}$ and PV material power conversion efficiency was $25 \%$ (Steinsiek et al., 2003). Japan's Kinki University chose the output power of $200 \mathrm{~W}$ with $808 \mathrm{~nm}$ wavelength LD to irradiate the PV materials on the kite fly machine. The PV materials were made of eight GaAs PV batteries with $28 \mathrm{~cm}^{2}$ each. The $0.017 \mathrm{~W}$ electrical power was gained from the unit area and incident photon-toelectron conversion efficiency was $21 \%$ (Kawashima et al., 2007). NASA put the silicon-based PV materials onto the space elevator, utilising the telescope system to transmit 1,030 nm wavelength and $8 \mathrm{~kW}$ power continuous laser, irradiating on the silicon-based PV materials to drive the space elevator. The silicon-based PV array which comprised 333 monocrystalline silicon $1 \mathrm{~m}^{2}$ chips was realising the power conversion efficiency of $35 \%$ (Balaguer and Abderrahim, 2008; Becker et al., 2013).

Single-junction PV materials have better power conversion efficiency. But they could only absorb one certain wavelength and afford the weaker brightness of incident light. The electrical power gained from the unit area is smaller. This is not conducive to reducing the size of the receiving device. To improve the overall photoelectric conversion efficiency of the material, considering a different type of PV materials could absorb a different certain wavelength of light, people combine different III-V materials according to different bandgap widths to gain the multijunction PV materials. This material can absorb many different wavelengths, to improve total power conversion efficiency. For InGaP/AsGa/Ga three-junction PV material, its photoelectric conversion efficiency is higher than that of ordinary materials. Still, efficiencies will need to be improved to accommodate LPT's evolution. The relevant research is already underway and mentioned in Han, (2018).

In 2000, Boeing-Spectrolab realised the conversion efficiency at $32.4 \%$, under 372 times the concentration. In the same year, the Shanghai space power supply institute, the ministry of information industry and other units have also carried out the corresponding research (Zhang et al., 2003; Wang and Zhang, 2012; Guo and Sun, 2007).

In 2006, Japan Kensuke Nishioka has studied the electricity character of InGaP/AsGa/Ga three-junction PV material which was changed due to the incident light brightness and temperature. In this lab, the open-circuit voltage, the short-circuit current, the fill factor and power conversion efficiency were concerned. The brightness range of the incident laser was $\sim 1$ to 200 times sunlight, and the material temperature range was $\sim 30$ to $240{ }^{\circ} \mathrm{C}$. The lab results showed that when the temperature stayed constant, with the incident laser brightness increased, the open-circuit voltage, the short-circuit current and power conversion efficiency would be increasing too. If the brightness stayed 
constant, with the temperature increased, the open circuit, the fill factor and the photovoltaic conversion efficiency were decreasing, but the short-circuit current was raising (Cotal et al., 2000; Nishioka et al., 2006).

In 2009, American researcher Geffrey et al. have studied the C1MJ and C2MJ three-junction GaAs materials' electrical property under the temperature range of $\sim 0$ to $120^{\circ} \mathrm{C}$ and incident light range of $\sim 1$ to 1,000 times sunlight. The result showed that the increase of the incident light brightness would be due to the fill factor and photovoltaic conversion efficiency first raises and then falls down. The temperature increase would cause the fill factor and photovoltaic conversion efficiency to decrease. When the temperature was constant, the peak was located at 200 times sunlight, and the photovoltaic conversion efficiency peak was under 500 times sunlight (Kinsey et al., 2009).

In 2010, the researchers at Hebei University of Technology have tested three-junction GaAs PV material to successfully gain significant electrical parameters. The experiment has measured the maximum photovoltaic conversion efficiency as $22.24 \%$, and the maximum output power was $23.56 \mathrm{~W}$, under the condition of 500 times sunlight. Also, once the temperature was rising $1^{\circ} \mathrm{C}$, the short-circuit current reduced to $1.9 \mathrm{~mA}$ (Nishioka et al., 2010).

In 2012, the research on the three-junction GaAs photovoltaic electrical parameters by Yunnan Normal University has shown that under irradiated by 676 times sunlight, the single three-junction GaAs material's shortcircuit current was 322 times theoretical result, the peak power was 316 larger than the theoretical value, the fill factor and material efficiency were reduced $4.6 \%$ and 3.9 , respectively, compared to the theoretical model (Zimmermann et al., 2015).

In 2013, the University of Shanghai for Science and Technology successfully built a three-junction GaAs material mathematic model and theoretically analysed the influence of the electrical characteristic by changing the incident light brightness and realistically measured the open-circuit voltage and material efficiency changing the rule. From the result, it is known that the increase of incident light brightness would aggrandise the opencircuit voltage and conversion efficiency. With the increase in temperature, the open-circuit voltage and conversion efficiency decreased (Yang et al., 2010).

In 2015, Henning Helmers et al. (2020) studied how the different temperatures or incident light brightness influenced the GaAs PV materials. During the experiment process, the temperature range was $\sim 5$ to $170{ }^{\circ} \mathrm{C}$ and the incident light brightness was $\sim 1$ to 3,000 times sunlight. The three-junction GaAs PV materials based on Ga would reduce efficiency under the high-temperature environment, as shown in the result (Wang et al., 2012).

In 2016, Hohn et al. researched under different temperatures as to how the laser wavelength influenced the three-junction GaAs PV materials. The result indicated that at a certain temperature, using a certain wavelength of the incident laser and irradiated by matched mixture lasers with the bandgap width of all the subdivision of the GaAs PV materials can improve the photovoltaic conversion efficiency (Wang et al., 2013).

In 2018, Han et al. from Nanjing University of Aeronautics and Astronautics studied different properties of incident lasers that influenced the efficiency of $\operatorname{InGaP} / \mathrm{GaAs} / \mathrm{Ga}$ three-junction PV materials. The experimental result indicated that with the property of 7:8:5 with the incident lasers' wavelength of $532 \mathrm{~nm}, 808 \mathrm{~nm}$, and $980 \mathrm{~nm}$, respectively. The power conversion efficiency was $33.549 \%$, which was the maximum value (Han, 2018).

Recently, in 2020, Kalyuzhnyy et al. studied the optimisation of InGaAs PV material, which could achieve the power conversion efficiency $>50 \%$, with the incident laser wavelength of 1,064 nm (Kalyuzhnyy et al., 2020).

Through the above research, from Table 4, it can be confirmed that GaAs material is the first choice of photovoltaic materials. It has the highest photoelectric conversion efficiency at present and has a small device size and convenient application. Moreover, the multi-junction GaAs material can adapt to multi-wavelength laser irradiation, thus reducing the requirements of the emitter and increasing the energy efficiency. The challenge of $\mathrm{PV}$ material is to increase the photovoltaic conversion efficiency and optimise the cell arrangement to reduce the internal power loss.

Table 4. Power conversion efficiency of different types of PV materials

\begin{tabular}{lccccc}
\hline PV materials & $\mathrm{Si}$ & Single-junction GaAs & Two-junction GaAs & Three-junction GaAs & Multi-junction GaAs \\
\hline \hline Efficiency (\%) & 23 & 27 & 30 & 38 & 50 \\
\hline
\end{tabular}

PV, photovoltaics. 


\section{Discussion}

After reading the previous literature, the author found two imperfections. One is that some application experiments only achieved the LPT to supply power to a certain application, and simply measured the parameters of the power transmission. The efficiency improvement of LPT was not further studied. Existing review articles have introduced the development of the overall system and each device in detail and listed some high-efficiency devices and methods to improve efficiency. However, the proposed devices and methods lack practical application cases, and their applications in complex environments have not been explored.

Compared to other WPT methods, LPT has outstanding advantages in motor drive. The CPT and IPT are called near-field WPTs, which have been widely used in the new energy source bus charging, mobile phone charging and embedded treating device charging, etc. In such fields, their power transmission efficiency is generally $>80 \%$. The devices of the emitter and receiver are accurately located at the stable position, which ensures transmission stability, accuracy and reliability at a high level. However, the transmission distance of CPT and IPT is too short to satisfy the requirements of long-distance power transmission applications. Usually, MPT and LPT are used in satellite charging, remote drone charging, etc. The efficiencies of MPT and LPT are both $<20 \%$, but the efficiency of MPT is lower than LPT. The devices of MPT are inconvenient to implement, which causes MPT to become less reliable and stable for use. But microwaves will not be influenced by media change such as different atmospheric conditions and the transmission accuracy could be better than LPT. LPT has a lower device size which is easy to implement. The reliability and stability will be at a medium level. But in practical applications, the emitter and receiver should be on a straight track and the route of the laser will be bent by air parameter change, which will cause the accuracy down. Considering the transmission efficiency, transmission distance and equipment suitability, the LPT is the most suitable wireless transmission method for motor drive. The comparison of the four wireless transmission methods is shown in Table 5.

The proposed LPT is also aimed at solving the problem of the limitation of wired energy transmission in harsh hazardous environments. The advantages of LPT are shown in Table 6 in detail. Taking the drive motor as an example, the author summarised a minimalist LPT model to achieve high-efficiency transmission applications. It includes an outer current supply, a LD, a PV material and a direct current (DC) motor as shown in Figure 4. The equivalent circuit of this model is shown in Figure 5. This LPT model plays a role in a DC/DC converter between

Table 5. Comparison of main parameters of four different types of WPT methods

\begin{tabular}{lllll}
\hline Method & CPT & IPT & MPT & LPT \\
\hline \hline Efficiency & High & High & Low & Low \\
Distance & Short & Medium & Long & Long \\
Convenience & High & Medium & Low & High \\
Complexity & Low & Medium & High & Low \\
Reliability & High & High & Low & Medium \\
Accuracy & High & High & Medium & Low \\
Stability & High & High & Medium & Medium \\
\hline
\end{tabular}

CPT, capacitive power transmission; IPT, inductive power transmission; LPT, laser power transmission; MPT, microwave power transmission; WPT, wireless power transmission.

Table 6. Comparison of wire and LPT in harsh hazardous application situations

\begin{tabular}{lll}
\hline Method & Wire & LPT \\
\hline \hline Efficiency & High & Low \\
High-temperature resistance & Low & High \\
Radiation resistance & Low & High \\
EMl & Exist & None \\
Safety & Low & High \\
\hline
\end{tabular}

EMI, electromagnetic interference; LPT, laser power transmission. 


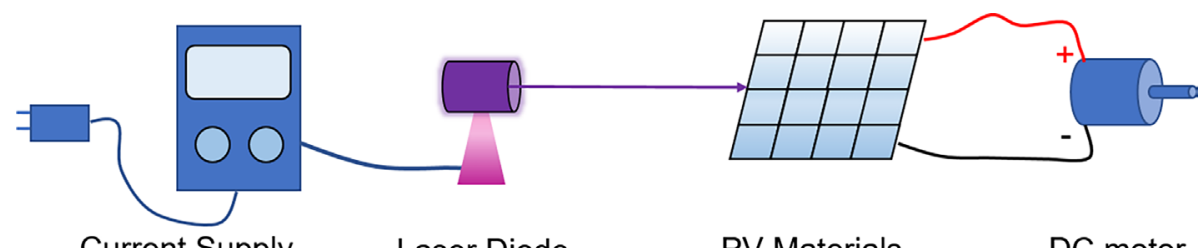

Current Supply Laser Diode $\quad$ PV Materials DC motor

Fig. 4. Proposed structure of laser power motor. PV, photovoltaics.

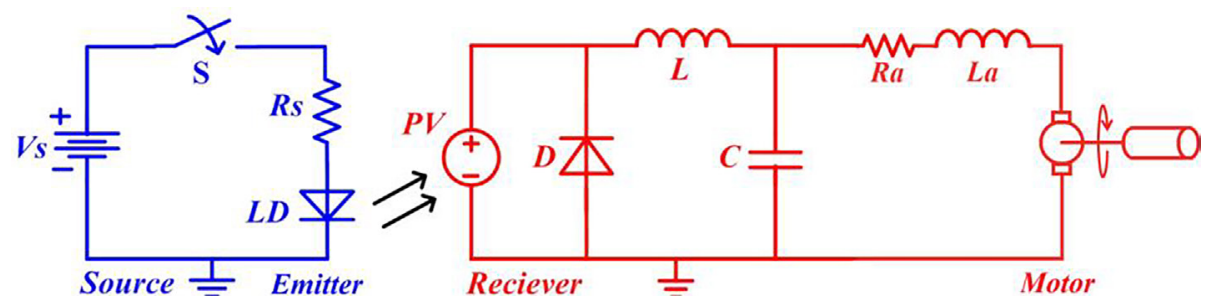

Fig. 5. Equivalent circuit of the laser power motor. LD, laser diode; PV, photovoltaics.

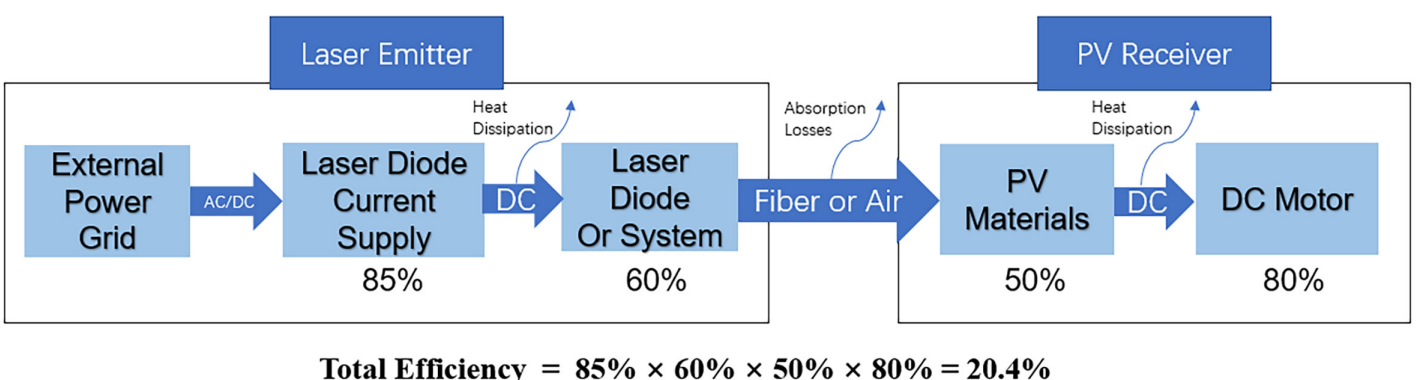

Fig. 6. Theoretical analysed efficiency of the motor drive by a laser-powered system. PV, photovoltaics.

current supply and motor load, so conversion efficiency is the first consideration. Choosing different LDs and PVs will have different usage conditions and overall efficiency. Judging from the results of the above review, the LD-GaAs is the advised LPT system for a laser power motor, because of the $810 \mathrm{~nm}$ best power efficiency of GaAs, which is suitable for LD's wavelength of $860 \mathrm{~nm}$. Both LD and GaAs PV materials are the most efficient equipment for energy conversion at present research. The transmission media is the atmosphere, which is a free space that is the most convenient choice. In the condition that the laser transmission route needs to be guided, the media should consider the optical fibre. If the application circumstances are high temperature or high radiation, such as nuclear station, the radiation resistance optical fibre can effectively reduce the environmental impact, as reviewed in Section 2.4.

The total power efficiency of the LPT system is analysed in Figure 6 . The power efficiency in a current power supply is generally $85 \%$. The power conversion efficiencies of LD and PV materials from the review result are ideally $60 \%$ and $50 \%$, respectively (Jin and Zhou, 2019). DC motor commonly has a power efficiency of about $80 \%$. The laser transfer process in media is dependent on the specific conditions, mainly on abortion rate and transmission distance. Theoretically, the total system delivery power efficiency is around $20.4 \%$, which ignores the transmission process in media space. This efficiency can achieve the goals required by the motor drive.

\section{Future Scope}

The research and development direction of LPT to achieve motor drive in future mainly has the following aspects: (1) improvement of device conversion efficiency, (2) LPT system's advanced control strategy and (3) the impact of LPT efficiency on complex environments and improvement methods. Through research progress in these directions, LPT will become a competitive energy transmission method and be widely used. 


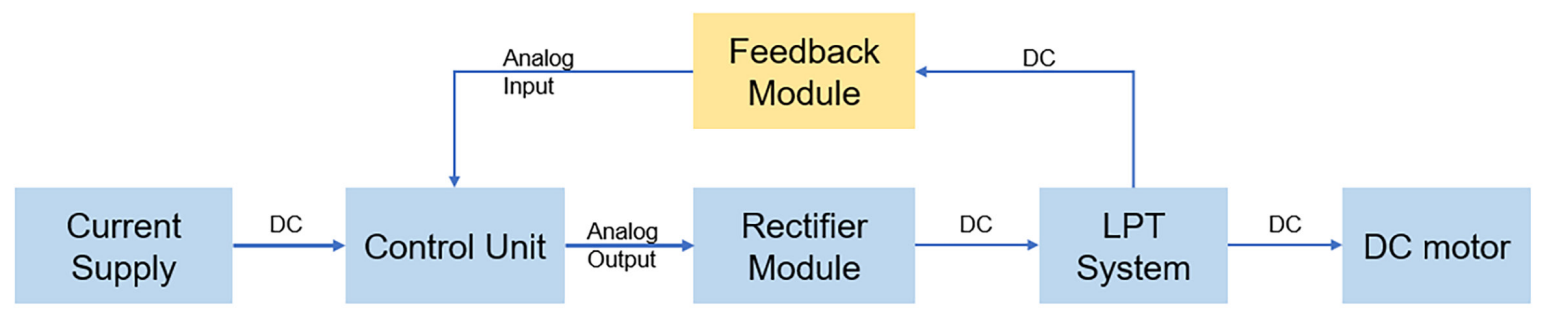

Fig. 7. Control strategy topology schematic structure of LPT. LPT, laser power transmission.

\subsection{Improvement of device conversion efficiency}

The limitation of LPT in practical applications is mostly due to the low transmission efficiency. Both LD and PV materials do not have the ideal high conversion efficiency. In order to get enough power for the load, two aspects are needed to improve. One is to develop new, more efficient devices of both laser and PV materials. The other is to optimise the operation of the equipment. For example, LD in a high-efficiency working state requires high accuracy and stability of the driving current. In addition to using a stable current source as much as possible, power electronic technology can also be used for rectification to ensure the quality of the LD's driving current. At present, there is little research on power electronics technology rectifying the LD's driving current. Moreover, the arrangement of the PV unit and the design of the internal circuit will also affect the overall output efficiency. Because the laser power beam density of LD is following the Gaussian distribution, the PV cells that receive insufficient light will become redundant internal resistance in the loop, reducing the conversion efficiency. Energy efficiency can be improved by optimising the arrangement of cells and adding electronic components.

\subsection{LPT system's advanced control strategy}

An effective and accurate control system strategy can not only improve the transmission efficiency but also meet the advanced requirements of the load such as the motor high-to-low speed and forward-to-reverse direction control. At the same time, it can also ensure stable output at the load end. Figure 7 shows the assumed control strategy schematic diagram and the topology of the structure proposed in Figure 4. A control unit uses the analogue input signal from the PV material terminal as the feedback of the LPT system output signal to control the analogue output signal to the rectifier module. The rectifier module is composed of power electronic devices and has the functions of stabilising and amplifying the driving current. The feedback signal module also plays a role in current modulation to adapt to the input requirements of the control unit. The design and applicability of power electronic technology for the demands of LPT is also a valuable research direction.

\subsection{Environmental interference improvement}

Some extremely harsh environments, such as nuclear stations and dusty industrial sites, will also have an impact on the LPT system's efficiency. How to eliminate the impact in these occasions and promote the advantages of LPT is also a direction worth exploring. The impact of the complex environment is concentrated on the transmission path. For example, the method to avoid the impact of strong nuclear radiation on atmospheric properties that change the transmission characteristics of lasers in the atmosphere. More advanced radiation-resistant optical fibres also need to be studied. The high-temperature resistance and radiation resistance of lasers and photovoltaic materials also need to be further studied and improved.

\section{Conclusion}

In this paper, an overall review of the LPT system development and existing application examples have been investigated. Existing LPT research has proved that applications powered by laser could be one feasible way. The limitation of this technology is mainly due to the transmission efficiency, which is, generally, around $20 \%$. The aspects of laser emitter, transmission media space, PV materials and the power conversion efficiency of the total LPT system are analysed. LD has a lower size, is easy to implement and use, has a good alignment of the beam and has high photovoltaic power conversion efficiency. The accuracy, stability and wave types will influence the 
working efficiency of LD, which could be one direction to improve the efficiency. If single LD is not enough for power demand, the diode laser system can be considered. But this technology will increase the complexity of the emitter device, which results in inconvenience in use. When the emitter and receiver are on a straight track and need not consider the transmission track, the atmosphere is the best media that saves a few steps of fibre installation and laser collimation. The atmosphere has different wavelength absorption rates, in order to have better transmission efficiency, and the chosen wavelength should be inside the range of the atmosphere's transmission window. Air transmission in short distances is convenient, but long-distance transmission will increase power loss and change the transmission route that reduces the accuracy. The optical fibre can also be used to build the transmission route. In a high-radiation environment, the transmission efficiency of fibre will be influenced. So, improving the radiation resistance of fibre is also important. GaAs has the best power conversion efficiency with current technology because its bandgap is moderate. Back, to select LD's wavelength, the best is around $850 \mathrm{~nm}$.

Then, compared to other WPT, LPT has a long transmission distance and it is easy to implement, which is a suitable method as an actuator for motor drive. The proposed structure of the LPT system for a motor drive is discussed in detail. This system adopts the high-efficiency equipment under the current technical level summarised by the review and evaluates the overall efficiency of the system. In the future scope, the development direction and expansion ideas of the system are listed in detail, and each aspect will help improve the efficiency and applicability, especially, the state that the system can reach under the optimisation by power electronics.

Finally, the proposed LPT to drive the motor is dedicated to avoid the limitations and losses in wired transmission, especially in harsh hazardous environments. From the efficiency of analysis, it can basically meet the needs of wireless transmission.

\section{References}

Alejnikov, V. S., Artjushenko, V. G., Belyaev, V. P., Vojtsekhovsky, V. V., Dianov, E. M., Lisitsky, I. S., Butvina, L. N., Masychev, V. I., Savenkova, T. N. and Sysoev, V. K. (1985). Fibre-Optic Cable for CO and CO2 Laser Power Transmission. Optics and Laser Technology, 17(4), pp. 213-214.

Anon. (2011). Rapid Development of Photovoltaic Technology. Dual-use Technology and Products, 11(3). Available at: https://kns.cnki.net/kcms/detail/ detail.aspx?dbcode $=$ CJFD\&dbname $=$ CJFD2011 \&filename $=$ HTJM201103007\&uniplatform $=$ NZKP $\mathrm{T} \& \mathrm{v}=\% 25 \mathrm{mmd} 2 \mathrm{Fd}$ 92uus1kgPPnbLzbkNe3pQJB ESOmY03XWhvUv88I8cvpiRf5bp1BO $\% 25 \mathrm{mmd}$ 2BJYNQLR\%25mmd2BUa

AUVSI. (2012). Laser Motive, Lockheed Demonstrate Real-World Laser Power. [Online]. Available at: https://www.flightglobal.com/news/articles/auvsilasermotive-lockheed-demonstrate-real-worldlaser-375166/

Balaguer, C. and Abderrahim, M. ed. (2008). Robotics and Automation in Construction. BoD-Books on Demand, pp. 373-380.

Becker, D. E., Chiang, R. and Keys, C. C. (2013). Photovoltaic Concentrator Based Power Beaming for Space Elevator Application. AIP Conference Proceedings. American Institute of Physics, 1230(1), pp. 271-281.
Becker, D. E., Chiang, R., Keys, C. C., Lyjak, A. W., Nees, J. A. and Starch, M. D. (2010). Photovoltaic Concentrator-Based Power Beaming for Space Elevator Application. AIP Conference Proceedings, 1230(1), pp. 271-281.

Billat, A., Blanc, J., Kuhnhenn, J. and Ricci, D. (2017). Photobleaching Effects in Multi-Mode Radiation Resistant Optical Fibers. 2017 17th European Conference on Radiation and its Effects on Components and Systems (RADECS), pp. 1-3.

Bogachev, A. V., Garanin, S. G., Dudov, A. M., Eroshenko, V. A., Kulikov, S. M., Mikaelian, G. T., Panarin, V. A., Pautov, V. O., Rus, A. V. and Sukharev, S. A. (2012). Diode Pumped Caesium Vapor Laser with Closed Cycle Laser Active Medium Circulation. Quantum Electronics, 42(2), pp. 95-98.

Brand, T., Unger, A., Koehler, B., Wolf, P., Beczkowiak, A. and Biesenbach, J. (2013). Diode Laser Platform for Multi-kW Applications. 2013 High Power Diode Lasers and Systems Conference (HPD), pp. 32-33.

Bull, S., Kaunga-Nyirenda, S. N. and Larkins, E. C. (2013). Design Considerations for High-Power External Cavity Laser Diodes. 2013 High Power Diode Lasers and Systems Conference (HPD), pp. 6-7.

Cotal, H. L., Lillington, D. R., Ermer, J. H., King, R. R., Karam, N. H., Kurtz, S. R., Friedman, D. J., Olson, J. M., Ward, S., Duda, A. and Emery, K. A. (2000). 
Highly Efficient 32.3\% Monolithic GalnP/GaAs/Ge Triple Junction Concentrator Solar Cells. National Renewable Energy Lab.

Guo, A. and Sun, Q. (2007). Progress of Semiconductor Solar Cell Technology Based on GaAs. Chinese Journal of Power Sources, 31(9), pp. 757-758.

Han, M. (2018). Study on Photoelectric Conversion Efficiency of GaAs Concentrator Cells Under Laser Irradiation. Nanjing University of Aeronautics and Astronautics. Available at: https://kns.cnki. net/kcms/detail/detail.aspx?dbcode $=$ CMFD\&d bname $=$ CMFD201901\&filename $=1019800556$. nh\&uniplatform $=N Z K P T \& v=s \% 25 m m d 2 F i H$ MT7eJYbThD4OCb\%25mmd2BMLIncq0HXd eU9F7R2QzvnVxHVzxWYMSpjhbuYNccxBuo3

Helal, M. A., Bull, S., Kaunga-Nyirenda, S. N., Lim, J. J. and Larkins, E. C. (2013). Simulation of HighBrightness Diode Lasers with Optical Feedback from Modules and Systems. 2013 High Power Diode Lasers and Systems Conference (HPD), pp. 14-15.

Helmers, H., Armbruster, C., von Ravenstein, M., Derix, D. and Schoner, C. (2020). 6-W Optical Power Link With Integrated Optical Data Transmission. IEEE Transactions on Power Electronics, 35(8), pp. 7904-7909.

Hengesbach, S., Witte, U., Traub, M. and Hoffmann, D. (2013). Design of a DFB/DBR Diode Laser Module Including Spectral Multiplexing Based on VBGs. 2013 High Power Diode Lasers and Systems Conference (HPD), pp. 16-17.

Huang, H. (2013). Theoretical and Experimental Study on Feedback Resonant Laser Energy Transmission. Doctor's Thesis. Tsinghua University.

Huang, R. K., Samson, B., Chann, B., Lochman, B. and Tayebati, P. (2015). Recent Progress on HighBrightness kW-Class Direct Diode Lasers. 2015 IEEE High Power Diode Lasers and Systems Conference (HPD), pp. 29-30.

Jin, K. and Zhou, W. (2019). Wireless Laser Power Transmission: A Review of Recent Progress. IEEE Transactions on Power Electronics, 34(4), pp. 3842-3859.

Kalyuzhnyy, N. A., Emelyanov, V. M., Evstropov, V. V., Mintairov, S. A., Mintairov, M. A., Nahimovich, M. V., Salii, R. A. and Shvarts, M. Z. (2020). Optimization of Photoelectric Parameters of InGaAs Metamorphic Laser $(\lambda=1064 \mathrm{~nm})$ Power Converters with Over 50\% Efficiency. Solar Energy Materials and Solar Cells, 217, pp. 110710.

Kawashima, N. and Takeda, K. (2005). Laser Energy Transmission for a Wireless Energy Supply to
Robots. Robotics and Automation in Construction, 10, pp. 373-380.

Kawashima, N., Takeda, K. and Yabe, K. (2007). Application of the Laser Energy Transmission Technology to Drive a Small Airplane. Chinese Optics Letters, 5(101), pp. S109-S110.

Kinsey, G. S., Hebert, P., Barbour, K. E., Krut, D. D., Cotal, H. L., Sherif, R. A. (2009). Concentrator Multijunction Solar Cell Characteristics Under Variable Intensity and Temperature. Progress in Photovoltaics: Research and Applications, 16(6), pp. 503-508.

Kleine, K. and Balu, P. (2017). High-Power Diode Laser Sources for Materials Processing. 2017 IEEE High Power Diode Lasers and Systems Conference (HPD), pp. 3-4.

Krasnoshchoka, A., Xu, J., Thorseth, A., Dam-Hansen, C., Jensen, O. B. (2019). High Luminous Flux Laser Lighting Using Single-Crystal Ce:YAG Phosphor. 2019 IEEE High Power Diode Lasers and Systems Conference (HPD), pp. 31-32.

Liu, F. (2016). Xie Honggang. Effect of y-radiation on optical fiber dispersion. Infrared and Laser Engineering, 45(1), pp. 116-121.

Lucas-Leclin, G., Schimmel, G., Albrodt, P., Hanna, M. and Georges, P. (2017). Coherent Combining Architectures for High-Brightness Laser Diodes. 2017 IEEE High Power Diode Lasers and Systems Conference (HPD), pp. 49-50.

McComb, T. S., Sims, R. A., Willis, C. C., Kadwani, P., Shah, L. and Richardson, M. (2010). Atmospheric Transmission Testing Using a Portable, Tunable, High Power Thulium Fiber Laser System. CLEO/QELS: 2010 Laser Science to Photonic Applications, pp. 1-2.

McCormick, D., Irwin, D., Stapleton, D., Braker, J., Koenning, T. and Patterson, S. (2015). UltraNarrow Spectral Linewidth Diode Lasers for the Pumping of Alkalis. 2015 IEEE High Power Diode Lasers and Systems Conference (HPD), pp. 25-26.

Mohammadnia, A., Ziapour, B. M., Ghaebi, H. and Khooban, M.H. (2021). Feasibility Assessment of Next-Generation Drones Powering by LaserBased Wireless Power Transfer. Optics and Laser Technology, 143, pp. 107283.

Nishioka, K., Sueto, T., Uchina, M. and Ota, Y. (2010). Detailed Analysis of Temperature Characteristics of an InGaP/InGaAs/Ge Triple-Junction Solar Cell. Journal of Electronic Materials, 39(6), pp. 704-708.

Nishioka, K., Takamoto, T., Agui, T., Kaneiwa, M., Uraoka, Y. and Fuyuki, T. (2006). Annual Output Estimation of Concentrator Photovoltaic Systems 
Using High-Efficiency InGaP/InGaAs/Ge TripleJunction Solar Cells Based on Experimental Solar Cell's Characteristics and Field-Test Meteorological Data. Solar Energy Materials and Solar Cells, 90(1), pp. 57-67.

Raible, D. E. (2008). High Intensity Laser Power Beaming for Wireless Power Transmission. Master's Thesis, Department of Electrical and Computer Engineering, Cleveland State University, Cleveland, $\mathrm{OH}, 5$.

Raible, D. E. (2011). Free Space Optical Communications with High Intensity Laser Power Beaming. Doctor's Thesis, Department of Electrical and Computer Engineering, Cleveland state University, Cleveland, $\mathrm{OH}, 6$.

Reng, N. and Beck, T. (1993). Transmission Properties of All-Silica Fibres for High-Power Nd: YAG Lasers. Optics and Laser Technology, 25(2), pp. 117-124.

Röhner, M., Wagner, L., Pietrzak, A. and Hülsewede, R. (2013). Fiber-Coupled High-Power Diode-Lasers with Highest Radiance. 2013 High Power Diode Lasers and Systems Conference (HPD), pp. 36-37.

Rossin, V., Peters, M., Demir, A., Morehead, J. J., Guo, J., Xiao, Y., Cheng, J., Hsieh, A., Duesterberg, R. and Skidmore, J. (2015). High Power, High Brightness Diode Lasers for kW Lasers Systems. 2015 IEEE High Power Diode Lasers and Systems Conference (HPD), pp. 35-36.

Rubenchik, A. M., Fedoruk, M. P. and Turitsyn, S. K. (2009). Laser Beam Self-Focusing in the Atmosphere. Physical Review Letters, 102(23), pp. 233902.

Sahai, A. and Graham, D. (2011). Optical Wireless Power Transmission at Long Wavelengths. 2011 International Conference on Space Optical Systems and Applications, pp. 164-170.

Shi, D., Zhang, L., Ma, H., Wang, Z., Wang, Y. and Cui, Z. (2016). Research on Wireless Power Transmission System Between Satellites. 2016 IEEE Wireless Power Transfer Conference (WPTC), pp. 1-4.

Song, Z. (2018). Energy Transfer Technology of $975 \mathrm{~nm}$ Fiber Coupled Semiconductor Laser. Space Electronic Technology, 15(2), pp. 101-105.

Steinsiek, F., Foth, W. P. and Weber, K. H. (2003). Wireless Power Transmission Experiment as an Early Contribution to Planetary Exploration Mission. Bremen: the 54th International Astronautical Congress, 3, pp. 169-176.

Summerer, L. and Purcell, O. (2009). Concepts for Wireless Energy Transmission via Laser. Europeans Space Agency (ESA)-Advanced Concepts Team.
Sumpf, B. (2016). Wavelength Stabilized High-Power Diode Lasers - Devices and Applications. 2016 International Conference Laser Optics (LO), pp. R3-3.

Wang, H. and Jiang, D. (2017). Design of High Temperature Gate Driver for SiC MOSFET for EV Motor Drives. 2017 IEEE Transportation Electrification Conference and Expo, Asia-Pacific (ITEC Asia-Pacific, pp. 1-6.

Wang, N. (2011). Research on the Key Technology of Laser Active Power Supply in Wireless Sensor Networks. Chongqing University. Available at: https://kns.cnki.net/kcms/detail/detail.aspx? $\mathrm{dbcode}=$ CDFD \&dbname $=$ CDFD 1214\&filena $m e=1012008792$.nh\&uniplatform $=$ NZKPT\&v=upB XyQhlld8gcVYbV32d\%25mmd2BP\%25mmd2F3K IMCwre\%25mmd2Bp5GNwtWS3MTW7ZEQ4gBC A4ZeOL723TBE

Wang, W., Li, M. and Ji, X. (2012). A Novel Fresnel Concentrator for GaAs Cells. Acta Optica Sinica, 7, pp. 198-204.

Wang, X. and Zhang, Y. (2012). Research Status and Development Trends of GaAs Trijunction Solar Cells. National MOCVD Academic Conference.

Wang, Z., Zhang, H. and Liu, Y. (2013). Theoretical and Experimental Analysis of Electrical Characteristics of InGaP/GaAs/Ge Three-junction GaAs Photovoltaic Cells. Proceedings of the CSEE, 33(27), pp. 168-174.

Wen, J. (2020). Fabrication and Properties of Radiation Resistant Polarization Keeping Fiber. Flight Control and Detection. 3(02), pp. 81-85.

Witte, U., Hamann, M., Di Meo, A., Rubel, D., Traub, M. and Hoffmann, D. (2015). High Power Diode Laser with 23W ex 35 um Fibre. 2015 IEEE High Power Diode Lasers and Systems Conference (HPD), pp. 17-18.

Witte, U., Hengesbach, S., Traub, M., Strotkamp, M., Jungbluth, B. and Hoffmann, D. (2013). High Brightness Diode Laser Module in the Red Spectral Range for Pumping Applications. 2013 High Power Diode Lasers and Systems Conference (HPD), pp. 30-31.

Xiao, B. (2017). Design and Implementation of Low Power Intelligent Fiber Energy Transmission System. Nanjing University of Posts and Telecommunications. Available at: https://kns.cnki. net $/ \mathrm{kcms} /$ detail/detail.aspx?dbcode=CMFD\&dbna me $=$ CMFD201801\&filename $=1017859792$.nh\&un iplatform=NZKPT\&v=K4QceUFOQkJyequ4S32Eh IA9ly5qfHzrBmMM1Y2j6Y7FUiWnMWTto\%25mm d2F365\%25mmd2Fa9KXdS 
Yang, Y., Chen, G. and Guo, L. (2010). Study on I-V Characteristics of Three-junction GaAs Solar Cells. Semiconductor Technology, 35(5), pp. 423-426.

Yao, Y. (2009). Study on Thermal Halo Effect of High Power Laser Propagation in Atmosphere. Xidian University.

Yugami, H., Kanamor, Y., Arashi, H., Niino, M., Moro, A., Eguchi, K., Okada, Y. and Endo, A. (1997). Field Experiment of Laser Energy Transimission and Laser to Electric Conversion. Honolulu: Proceedings of the Intersociety Energy Conversion Engineering Conference, 1, pp. 625-630.

Zhang, G. X., Chen, S., Xu, S. G., Luo, B. and Zhao, Y. M. (2010). Application and Research of Laser De-icing in Power System. 2010 IEEE International Power Modulator and High Voltage Conference, pp. 470-473.

Zhang, M. (2017). Experimental Study on Gamma Irradiation Effect of Double Cladding Ultraviolet Fiber. Atomic Energy Science and Technology, 51(3), pp. 536-542.

Zhang, Q., Yun, F. and He, Q. (2020). Research on Space Radiation Damage Mechanism and Performance Improvement of Quartz Fiber. Fiber and Cable and Its Application Technology, 6, pp. 7-9.

Zhang, Z., Lu, J. and Chi, W. (2003). Progress and Prospect of GaAs Solar Cell Technology. Shanghai Aerospace, 20(3), pp. 33-38.

Zhao, X. (2007). Study on the Energy Transmission Characteristics of Fiber Laser with Peak Power. China Academy of Engineering Physics.

Zhou, W. (2018). Research on Key Technology of Laser Radio Energy Transmission System. Nanjing University of Aeronautics and Astronautics.

Zhou, W. and Jin, K. (2015a). Efficiency Evaluation of Laser Diode in Different Driving Modes for Wireless
Power Transmission. IEEE Transactions on Power Electronics, 30(11), pp. 6237-6244.

Zhou, W. and Jin, K. (2015b). Efficiency Optimization Inject Current Characteristic of Laser Diode for Wireless Power Transmission. 2015 IEEE Energy Conversion Congress and Exposition (ECCE), pp. 3082-3086.

Zhuang, Y., Hua, L. and Guo, B. (2014). Research and Design of Laser Energy Converter for Laser Energy Transmission System. Infrared, 35(12), pp. 35-40.

Zimer, H., Haas, M., Nagel, S., Ginter, M., Ried, S., Rauch, S., Killi, A. and Heinemann, S. (2015). Spectrally Stabilized and Combined Diode Lasers. 2015 IEEE High Power Diode Lasers and Systems Conference (HPD), pp. 31-32.

Zimer, H., Ried, S., Tillkorn, C., Killi, A., Barnowski, T., An, H. and Schmidt, B. (2013). Beam Combining Technologies for Direct Diode Laser Systems at the kW Power Level. 2013 High Power Diode Lasers and Systems Conference (HPD), pp. 38-39.

Zimmermann, S., Helmers, H., Tiwari, M. K., Paredes, S., Michel, B., Wiesenfarth, M., Bett, A. W. and Poulikakos, D. (2015). A High-Efficiency Hybrid High-Concentration Photovoltaic System. International Journal of Heat and Mass Transfer, 89, pp. 514-521.

Zotov, K. (2008a). Radiation-Resistant ErbiumDoped Fiber for Spacecraft Applications. IEEE Transactions on Nuclear Science, 55(4), pp. 2213-2215.

Zotov, K. V., Likhachev, M. E., Tomashuk, A. L., Kosolapov, A. F., Bubnov, M. M., Yashkov, M. V., Guryanov, A. N. and Dianov, E. M. (2008b). Radiation Resistant Er-Doped Fibers: Optimization of Pump Wavelength. IEEE Photonics Technology Letters, 20(17), pp. 1476-1478. 